\title{
Transparent Conducting Indium Zinc Tin Oxide Anode for Highly Efficient Phosphorescent Organic Light Emitting Diodes
}

\author{
Jung-Hyeok Bae, ${ }^{a}$ Jong-Min Moon, ${ }^{\text {a }}$ Soon Wook Jeong, ${ }^{\text {a Jang-Joo Kim, }}{ }^{\text {b }}$ \\ Jae-Wook Kang, ${ }^{\mathrm{c}}$ Do-Geun Kim, ${ }^{\mathrm{c}}$ Jong-Kuk Kim, ${ }^{\mathrm{c}}$ Jeong-Woo Park, ${ }^{\mathrm{d}}$ and \\ Han-Ki Kim ${ }^{\text {a,z }}$
}

${ }^{a}$ School of Advanced Materials and Systems Engineering, Kumoh National Institute of Technology, Gumi 730-701, Korea

${ }^{b}$ School of Materials Science and Engineering, Seoul National University and Organic Light Emitting Diodes Center, Sillim-dong, Seoul 151-741, Korea

${ }^{c}$ Surface Technology Research Center, Korea Institute of Machinery and Materials, Gyeongnam 641-831, Korea

${ }^{d}$ Department of Materials Science and Engineering, Gwangiu Institute of Science and Technology, Gwangju 500-712, Korea

The preparation and characteristics of a transparent conducting indium zinc tin oxide (IZTO) anode for highly efficient phosphorescent organic light emitting diodes (OLEDs) is described. The resistivity and transmittance of the IZTO anode are comparable to reference $\mathrm{In}_{2} \mathrm{O}_{3}$ (ITO) anode films even though it was prepared at room temperature. In addition, the work function of the ozone-treated amorphous IZTO anode $(5.12 \pm 0.02 \mathrm{eV})$ is much higher than that of ozone-treated reference ITO anodes $(4.94 \pm 0.02 \mathrm{eV})$. The current-voltage-luminance characteristics and efficiencies of OLEDs prepared on the IZTO anode are critically dependent on the sheet resistance of the IZTO anode. Furthermore, both the quantum efficiency and power efficiency of the OLED fabricated on the amorphous IZTO anode are much higher than those of an OLED with the reference ITO anode due to the higher work function of the IZTO anode than those of conventional ITO anode. This indicates that IZTO is an alternative material for conventional ITO anodes used in OLEDs and flexible displays.

(C) 2007 The Electrochemical Society. [DOI: 10.1149/1.2799745] All rights reserved.

Manuscript submitted June 12, 2007; revised manuscript received September 10, 2007. Available electronically October $29,2007$.

Organic light emitting diodes (OLEDs) are of considerable importance for their potential as a new generation of flat panel displays and flexible displays due to their high peak brightness, high dark room contrast, low power consumption, low-cost, super viewing ability, and fast response time. ${ }^{1,2}$ In order to obtain highperformance OLEDs and flexible OLEDs, it is very important to develop a high-quality anode layer with low resistance, high transparency, chemical stability, and a high work function. ${ }^{1,2}$ Although Sn-doped $\mathrm{In}_{2} \mathrm{O}_{3}$ (ITO) has been commonly used in OLEDs and flexible OLEDs as an anode material due to high conductivity and transmittance in visible range, the conventional ITO anode has several problems, such as an imperfect work function alignment, chemical instability, high process temperature, and easy deterioration of the ITO target. ${ }^{3,4}$ Recently, both ternary oxide and multicomponent oxides, such as $\mathrm{Al}-\mathrm{Zn}-\mathrm{O}$ (AZO), Ga-Zn-O (GZO), $\mathrm{Zr}-\mathrm{Zn}-\mathrm{O}$ (ZZO), In-Ga-O (IGO), In-Zn-O (IZO), In-Ga-Sn-O (IGTO), and Zn-In-Sn-O (IZTO) have gained much attention as promising anode materials for OLED and flexible OLEDs. ${ }^{3,5-7}$ Among the various multicomponent-TCO materials, IZTO films have recently been recognized as attractive anode materials in OLEDs and flexible OLEDs due to their high work function, good conductivity, high-transparency, and low-deposition temperature. ${ }^{3}$ Phillips et al. reported that $\mathrm{Zn}_{x} \mathrm{In}_{2-y} \mathrm{SnyO}_{3+x-\delta}$ film grown by pulsed laser deposition and sputtering has high conductivity and transmittance within ranges typically reported for ITO films. ${ }^{8}$ In addition, Ambrosini et al., investigating zinc doping in cosubstituted $\mathrm{In}_{2-2 x} \mathrm{Sn}_{x} \mathrm{Zn}_{x} \mathrm{O}_{3-\delta}$ bulk pellet, found that the solubility of $\mathrm{ZnO}$ and $\mathrm{SnO}_{2}$ in $\mathrm{In}_{2} \mathrm{O}_{3}$ can be greatly increased up to $x=0.4$ by codoping with equal amounts of $\mathrm{Zn}$ and $\mathrm{Sn}$ in the $\mathrm{In}_{2} \mathrm{O}_{3}$. ${ }^{9}$ Mark et al., in an investigation of the high work function transparent conducting oxide as an anode for an OLED, reported that an IZTO film has a higher work function $(\sim 6.1 \mathrm{eV})$ than a commercial ITO $(\sim 4.7 \mathrm{eV}))^{3,10}$ Although the electrical and optical characteristics of IZTO films have been reported, the use of amorphous IZTO anode in phosphorescent OLEDs has not yet been reported in detail. ${ }^{11-13}$

In this work, we report on the characteristics of an amorphous IZTO (a-IZTO) anode layer grown using radio frequency (rf) sput-

zz-mail: hkkim@kumoh.ac.kr tering at room temperature in a pure Ar ambient without the addition of reactive oxygen gas. We obtained high-quality a-IZTO anode films with high conductivity and transmittance comparable to conventional ITO anodes without intentional substrate heating, even though it was prepared at room temperature. Phosphorescent OLEDs prepared on this IZTO anode exhibited identical current density-voltage-luminescence $(J-V-L)$ characteristics as an OLED fabricated on a conventional ITO anode. In addition, both the quantum efficiency and power efficiency of OLEDs prepared on the a-IZTO anode are much higher than those of OLEDs prepared on conventional ITO anodes due to the high work function of the IZTO anode.

\section{Experimental}

IZTO anode films were deposited on a glass substrate using an rf sputtering system at room temperature under pure Ar ambient without the addition of reactive oxygen gas. A specially designed 3 in. IZTO target ( 5 wt $\% \mathrm{ZnO}$ and 5 wt $\% \mathrm{SnO}_{2}$ co-doped $\mathrm{In}_{2} \mathrm{O}_{3}$ ) was used as a target and was placed $100 \mathrm{~mm}$ from the glass substrate. At a constant Ar flow rate of $20 \mathrm{sccm}$, a working pressure of $5 \mathrm{mTorr}$ and deposition time of $20 \mathrm{~min}$, IZTO films were grown on a glass substrate with dimensions of $40 \times 40 \mathrm{~mm}$ as a function of $\mathrm{rf}$ power. For comparison, a reference ITO/glass sample (Asahiglass Fine Techno Co., Ltd.) was purchased, which is used in the OLED industry. The transmittance of the IZTO/glass and reference ITO/glass were measured in the wavelength range from 220 to $800 \mathrm{~nm}$ by a $\mathrm{UV} /$ visible spectrometer. Sheet resistance and the resistivity of the IZTO anode film were measured at room temperature by means of Hall effect measurement with van der Pauw geometry. To investigate the structural properties of the IZTO film, X-ray diffraction (XRD) and synchrotron X-ray scattering examinations were performed. The work functions of the IZTO and reference ITO anode films were measured by photoelectron spectroscopy with a UV source (PKI model AC-2) at atmospheric pressure after surface ozone treatment. The surface morphology of the IZTO and reference ITO anodes was analyzed by atomic force microscopy (AFM). To compare electrical and optical properties of OLED fabricated on IZTO and reference ITO anode, we prepared standard phosphorescent OLEDs using IZTO and ITO. After conventional wet cleaning and UV-ozone treatment for $10 \mathrm{~min}$, both the IZTO/glass 


\begin{tabular}{|c|c|c|c|c|}
\hline & $30 \mathrm{~W}$ & $40 \mathrm{~W}$ & $50 \mathrm{~W}$ & $60 \mathrm{~W}$ \\
\hline Thickness (nm) & 110 & 166 & 214 & 247 \\
\hline $\begin{array}{l}\text { Deposition rate } \\
(\mathrm{nm} / \mathrm{min})\end{array}$ & 5.50 & 8.31 & 10.70 & 12.35 \\
\hline $\begin{array}{l}\text { Sheet resistance } \\
(\Omega / \square)\end{array}$ & 37.02 & 23.07 & 15.56 & 20.63 \\
\hline $\begin{array}{l}\text { Transmittance (\%) at } \\
550 \mathrm{~nm}\end{array}$ & 67.46 & 74.8 & 91.30 & 83.6 \\
\hline
\end{tabular}

and reference ITO/glass samples were transferred to an organic/ metal evaporation system simultaneously. We used $40 \mathrm{~nm}$ thick $\alpha$-napthylphenlylbiphenyl $(\alpha-\mathrm{NPB})$ and $30 \mathrm{~nm}$ thick 4,4'-bis(9-carbazolyl)-biphenyl (CBP) doped with $6 \mathrm{wt} \%$ $\left[\operatorname{Ir}(\mathrm{ppy})_{3}\right]$ as a hole transport layer (HTL) and an emitting layer (EL), respectively. Subsequently, a $10 \mathrm{~nm}$ thick 2,9-dimethyl-4,7diphenyl-1,10-phenanthroline (bathocuproine: BCP) layer was grown on the EL layer as a hole blocking layer (HBL). A $40 \mathrm{~nm}$ thick tris-(8-hydroxyquinoline) aluminum $\left(\mathrm{Alq}_{3}\right)$ layer and a $1 \mathrm{~nm}$ $\mathrm{LiF}$ layer were then deposited as electron transport (ETL) and electron injection layers (EIL), respectively. Finally, a $100 \mathrm{~nm}$ thick Al cathode layer was patterned using a shadow metal mask. After deposition of the $\mathrm{Al}$ cathode, the OLED cells were glass encapsulated to protect the OLED cells from the moisture and the oxygen in air. Current density-voltage-luminance $(J-V-L)$ characteristics of phosphorescent OLEDs fabricated on different anode materials were measured with a Keithley 2400 and a Si photodiode mounted below the OLEDs.

\section{Results and Discussion}

Table I shows the thickness and deposition rate of the IZTO films as a function of rf power at constant flow of pure $\operatorname{Ar}(20 \mathrm{sccm})$, working pressure of $5 \mathrm{mTorr}$, and deposition time of $20 \mathrm{~min}$ to determine the optimum electrical and optical properties of the IZTO films. Both thickness and deposition rate of the IZTO films monotonically increased with increasing rf power due to increase of the plasma density. In general, the sputtering rate $S_{\mathrm{r}}$ (in nanometers per minute) can be expressed as ${ }^{14}$

$$
S_{\mathrm{r}}=S_{\text {ions }}+S_{\text {neutrals }}
$$

where $S_{\text {ions }}$ and $S_{\text {neutral }}$ are the sputtering rate of Ar ions and neutrals, respectively. In particular, the $S_{\text {ions }}$ is as follows

$$
S_{\text {ions }}=\frac{6.23 J W\langle Y\rangle}{\rho}
$$

where $W$ and $\rho$ are the atomic weight (in grams per molecule) and density (in grams per cubic centimeter) of sputtered atoms, $J$ is the current density at the target (microamperes per centimeter squared) and $\langle Y\rangle$ is the mean sputtered yield. This equation indicates that the sputtering rate of Ar ions is critically dependent on the current density, which is applied on the cathode target. Therefore, the dependence of IZTO deposition rate on the rf power can be explained by the dependence of the $S_{\text {ions }}$ on the current density.

Figure 1 shows the sheet resistance and resistivity of the IZTO anode layer as a function of rf power at a constant Ar flow rate of $20 \mathrm{sccm}$, a working pressure of $5 \mathrm{mTorr}$, and deposition time of $20 \mathrm{~min}$. Note that the sheet resistance and resistivity of the IZTO anode dramatically decreased with an increase in rf power. The minimum sheet resistance of $15.5 \Omega / \square$ and resistivity of 3.8 $\times 10^{-4} \Omega \mathrm{cm}$ was obtained using an $\mathrm{rf}$ power of $50 \mathrm{~W}(214 \mathrm{~nm}$ thick IZTO) under pure Ar at room temperature. The decrease in sheet resistance and resistivity in the IZTO films with increasing rf power can be attributed to the increase in the density of the IZTO

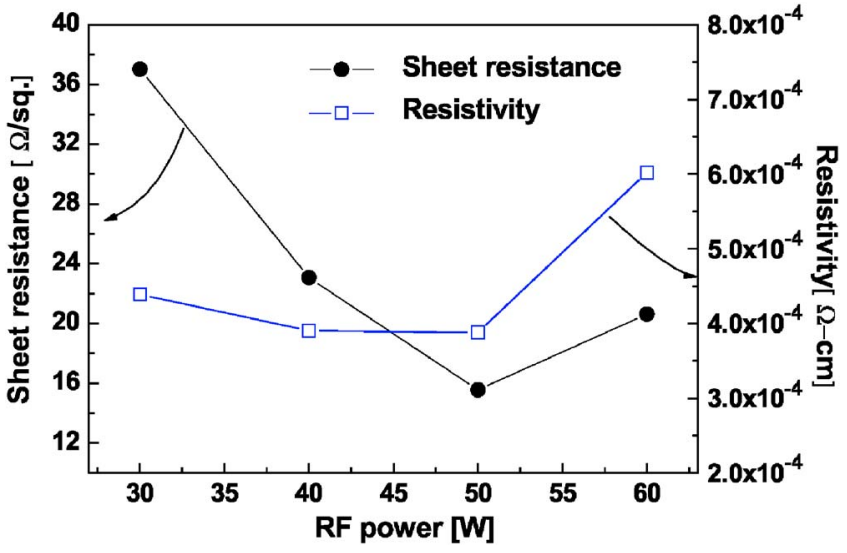

Figure 1. (Color online) Sheet resistance and resistivity of IZTO anode films grown on a glass substrate as a function of rf power under pure Ar ambient without the addition of reactive oxygen gas.

anode. Pan et al. reported that the decrease in resistivity of IZO films with increasing rf power is related to an increase in the density of the IZO film and the elimination of phonon scattering and ionized impurity scattering. ${ }^{15}$ However, a further increase in rf power leads to an increase in the sheet resistance and resistivity of the IZTO film due to the change in the stoichiometry in the IZTO anode. Although it was prepared at room temperature, the IZTO anode film showed comparable sheet resistance and resistivity to conventional ITO anodes prepared at high substrate temperatures in the range of $200-300^{\circ} \mathrm{C}^{4,16}$ This indicates the possibility of an IZTO film as a promising anode material for OLEDs and flexible OLEDs.

Figure 2 shows the optical transmittance spectra of the IZTO layer as a function of rf power and reference ITO anode films on a glass substrate while the picture inset compares the IZTO and ITO anodes. Without intentional substrate heating, postannealing process, or addition of reactive oxygen gas, transparent IZTO anodes can be obtained under pure Ar at ambient temperature. We found that the optical transmittance of the IZTO film in the green region significantly increased with increasing rf power from 30 to $50 \mathrm{~W}$. However, further increase of rf power up to $60 \mathrm{~W}$ leads to a slight decrease in transmittance of the IZTO film due to change of stoichiometry as expected from resistivity of IZTO film shown in Fig. 1.

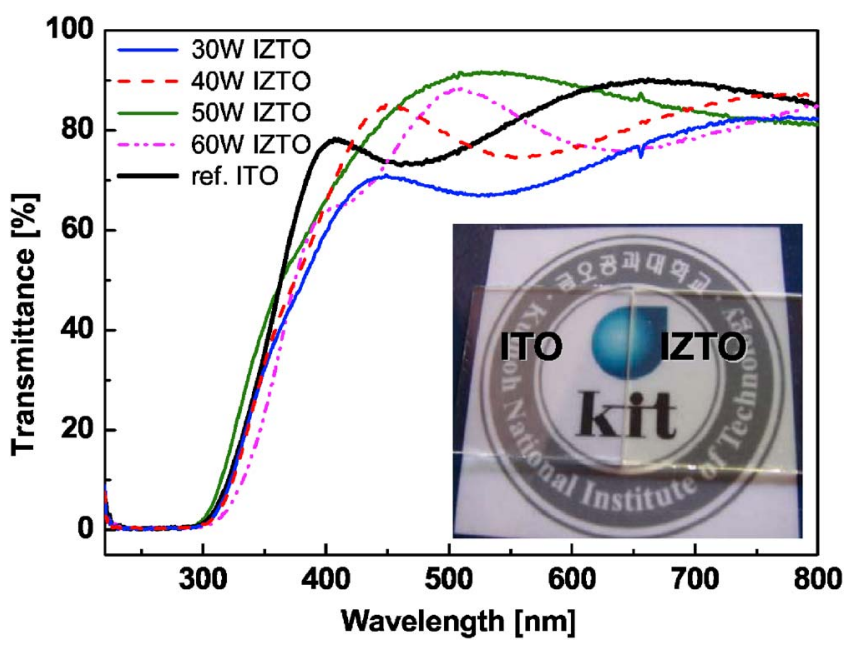

Figure 2. (Color online) Comparison of optical transmittance spectra obtained from IZTO $(30,40,50$, and $60 \mathrm{~W}$ rf power) and a reference ITO anode film with the inset of a visual comparison of the IZTO $(50 \mathrm{~W})$ and reference ITO anode. 

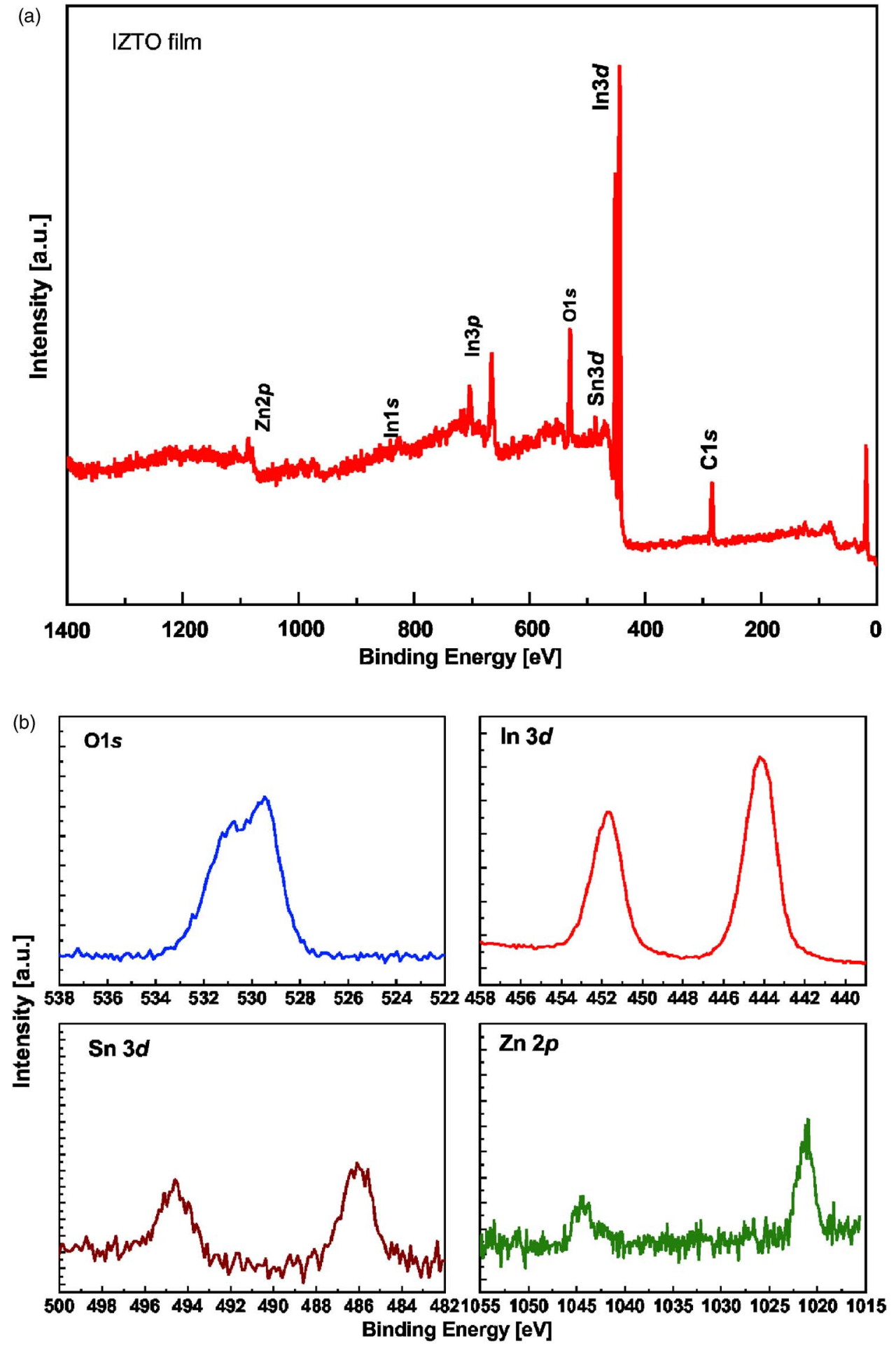

Figure 3. (Color online) (a) XPS wide scan and (b) core level spectra of O 1s, In $3 \mathrm{~d}, \mathrm{Sn} 3 \mathrm{~d}$, and $\mathrm{Zn} 2 \mathrm{p}$ for the IZTO film grown at rf power of $50 \mathrm{~W}$.
Note that the transmittance of the IZTO films $(50 \mathrm{~W}$ and $60 \mathrm{~W}$ samples) in the green region is much higher than that of the reference ITO films. Although the transmittance of the IZTO anode film in the UV region is lower than that of the reference ITO anode films, the average optical transmittance $(\sim 91.3 \%)$ of the IZTO anode (50 W sample) in the green region between 500 and $550 \mathrm{~nm}$ is much higher than that of a reference ITO (84.5\%) sample. The inset of Fig. 2 shows that IZTO anodes grown at $50 \mathrm{~W}$ rf power have a transparency comparable to reference ITO anode.

Figure 3 shows XPS wide scan and core level spectra obtained from the surface of the IZTO anode film grown at optimized condi- tion $(50 \mathrm{~W}$ rf power). The XPS wide scan and core level spectra data shows $\mathrm{O} 1 \mathrm{~s}$, In 3d, Zn 2p, and Sn 3d peaks, which are indicative of a bare IZTO anode layer. Compared to O $1 \mathrm{~s}$ and In $3 \mathrm{~d}$ peaks, the $\mathrm{Sn} 3 \mathrm{~d}$ and $\mathrm{Zn} 2 \mathrm{p}$ peaks show weaker peak intensity due to low doping concentration of $\mathrm{ZnO}(5 \mathrm{wt} \%)$ and $\mathrm{SnO}_{2}(5 \mathrm{wt} \%)$ in $\mathrm{In}_{2} \mathrm{O}_{3}$. Although Phillips et al. suggested that near stoichiometry IZTO film such as $\mathrm{Zn}(\mathrm{In}, \mathrm{Sn})_{2} \mathrm{O}_{4}$ or $\mathrm{Zn}_{3}(\mathrm{In}, \mathrm{Sn})_{5} \mathrm{O}_{10.5}$ with spinel structure have a high conductivity, we could obtain high conductivity from the IZTO film with low concentration of $\mathrm{ZnO}$ and $\mathrm{SnO}_{2}$ dopants. ${ }^{8}$ This is because the dominant component in the conductiv- 


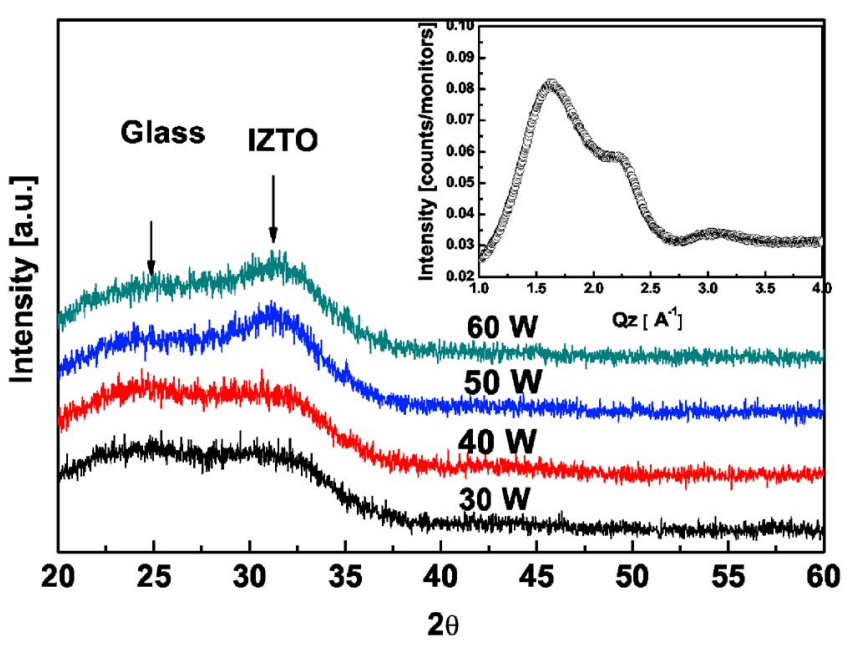

Figure 4. (Color online) XRD plots obtained from an IZTO anode film as a function of $\mathrm{rf}$ power with the inset of synchrotron X-ray scattering result of optimized IZTO anode film (rf $50 \mathrm{~W}$ ).

ity of IZTO film is stable oxygen vacancies. ${ }^{9,17}$ The two binding energies of 528.8 and $530.8 \mathrm{eV}$ in $\mathrm{O} 1 \mathrm{~s}$ core level spectra are caused by the two types of $\mathrm{O}^{2-}$ ions in the IZTO anode layer. The lower binding energy peak $\left(\mathrm{O}_{\text {II }}\right)$ is from the $\mathrm{O}^{2-}$ ions, which have neighboring indium atoms with their full complement, and the higher binding energy peak $\left(\mathrm{O}_{\mathrm{I}}\right)$ corresponds to oxygen-deficient regions. ${ }^{18,19}$

Figure 4 shows the XRD plots obtained from the IZTO anode films as a function of rf power with inset of synchrotron X-ray scattering result of optimized IZTO film (50 W rf power). Regardless of rf power, all XRD plots of the IZTO anode films in Fig. 4 show weak and broad peaks representing amorphous glass $\left(\sim 23^{\circ}\right)$ and amorphous IZTO layer $\left(\sim 31^{\circ}\right)$. Because of the low substrate temperature during sputtering process, all IZTO films show amorphous structure. In general, crystallization of ITO film occurs rapidly at low substrate temperature due to low amorphous/crystalline transition temperature $\left(T / T_{\mathrm{m}}<0.19-150^{\circ} \mathrm{C}\right)$. However, $\mathrm{ZnO}$ doped $\mathrm{In}_{2} \mathrm{O}_{3}$ could maintain a stable amorphous structure below $500^{\circ} \mathrm{C}$ due to high amorphous/crystalline transition temperature $\left(\sim 500^{\circ} \mathrm{C}\right){ }^{20}$ In the case of the IZTO film, the immiscibility of $\mathrm{ZnO}$ and $\mathrm{SnO}_{2}$ in $\mathrm{In}_{2} \mathrm{O}_{3}$ also leads to a stable amorphous structure as expected from IZO film. To make crystalline IZTO films, phase separation of $\mathrm{ZnO}$ and $\mathrm{SnO}_{2}$ from $\mathrm{In}_{2} \mathrm{O}_{3}$ is necessary. However, the kinetics of phase separation of $\mathrm{ZnO}$ and $\mathrm{SnO}_{2}$ in IZTO film are very low; as a result, the IZTO can maintain a more stable amorphous structure than conventional ITO film. This structural stability of the amorphous IZTO films is beneficial for the stable performance of OLEDs and flexible OLEDs.

Figure 5 shows surface scanning electron microscopy (SEM) images of the reference ITO and optimized IZTO anode film grown on the glass substrate. It is shown that the reference ITO anode in Fig. 5 a has a relatively rough surface due to preferred orientation of the ITO. ${ }^{4}$ However, thus far the IZTO anode film grown at room temperature in Fig. 5b, the surface is very smooth with very fine grains. In addition, the surface morphology of the IZTO anode (rms roughness: $0.237 \mathrm{~nm}$ ) grown at $50 \mathrm{~W}$ rf power is much smoother than that of the reference ITO anode (rms roughness: $3.964 \mathrm{~nm}$ ) due to the low preparation temperature and high stability of IZTO materials. The smooth surface of the anode layer is very important in OLEDs and flexible OLEDs because anode spikes can cause breakdown or shorting of OLEDs. ${ }^{1,2}$

Figure 6 shows chemical structures of molecules used in a phosphorescent OLED and device structure. To compare electrical and optical properties of the phosphorescent OLEDs fabricated on refer-
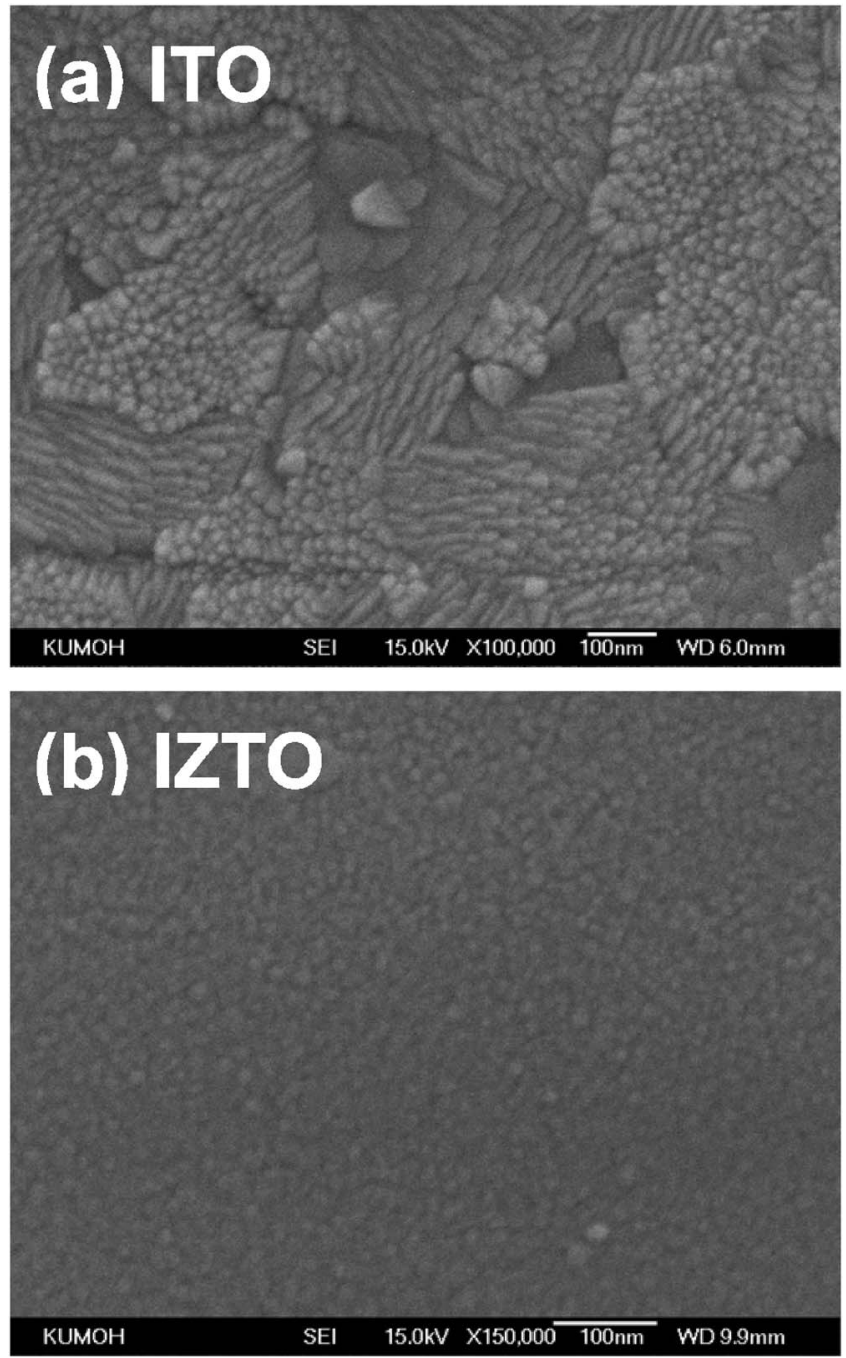

Figure 5. Surface SEM images of (a) reference ITO and (b) optimized amorphous IZTO anode films grown at rf power of $50 \mathrm{~W}$.

ence ITO and IZTO anode, all organic films and cathode layer above anode layers were simultaneously deposited on reference ITO and IZTO anode, respectively. To enhance external quantum efficiency (EQE) and power efficiency (PE) of the OLED, a $\operatorname{Ir}[\mathrm{ppy}]_{3}$-doped CBP layer was employed as EM layer. ${ }^{21,22}$

Figure 7 a shows the $J-V$ characteristics of an OLED fabricated on the IZTO and the reference ITO anode films, respectively. It was shown that the $J-V$ characteristics of an OLED with a IZTO anode critically depend on the sheet resistance of the IZTO anode layers. ${ }^{7}$ In addition, the $J-V$ characteristics in the log-log plot appear to follow power law $\left(J \propto V^{m}\right)$, where $m$ varies from 1 to 2 at low voltage and from 9 to 11 at high voltage. ${ }^{23}$ The OLED fabricated on the lower sheet resistance of IZTO shows lower turn-on voltage and higher current density. In particular, the OLEDs fabricated on a-IZTO with a sheet resistance of $37.02 \Omega / \square$ exhibit severe leakage current before being turned on. However, OLEDs fabricated on a-IZTO with a sheet resistance below $\sim 23 \Omega / \square$ show an identical $J-V-L$ curve to the reference OLED with a reference ITO anode. However, it was shown that the current density of the OLED fabricated on a-IZTO was slightly lower than that of the OLED fabricated on reference ITO at high voltage region. This could be attributed to the difference in trap densities of organic layers, which was grown on the different a-IZTO and ITO anode materials. It was reported that current density in ohmic regime at low voltage is determined by the anode contact properties rather than organic bulk 


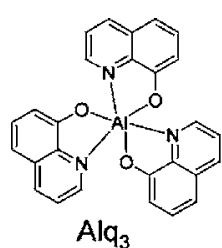

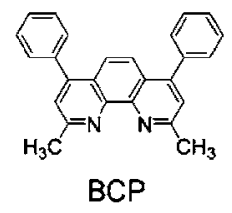

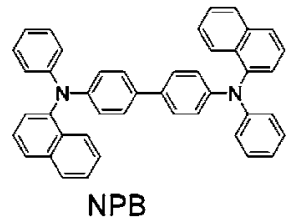

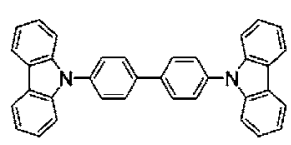

CBP

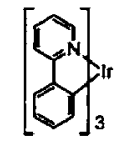

$\operatorname{Ir}(\mathrm{ppy})_{3}$

\begin{tabular}{|c|}
\hline Al $(100 \mathrm{~nm})$ \\
\hline LiF $(1 \mathrm{~nm})$ \\
\hline Alq $_{3}(40 \mathrm{~nm})$ \\
\hline BCP $(10 \mathrm{~nm})$ \\
\hline CBP-Ir $[\text { [Ppy }]_{3}(30 \mathrm{~nm})$ \\
\hline NPB $(40 \mathrm{~nm})$ \\
\hline IZTO or ITO \\
\hline Glass \\
\hline
\end{tabular}

Figure 6. Molecular structure of HIL, HTL, EL, HBL, and ETL layers used in a phosphorescent OLED and schematic device structure of a phosphorescent OLED.

properties. ${ }^{23-26}$ However, at the high-voltage region after the onset of electroluminance, the current density could be governed by the density and distribution of traps in the bandgap of organic materials. ${ }^{26}$ Therefore, the difference of trap densities in the organic layers could result in the increased current density of the OLED with reference ITO anode at the high-voltage region. The $I-V$ curves of the OLEDs with different IZTO and reference ITO anodes also show a similar steep increase after onset as shown in Fig. 7b. Despite similar $J-V$ behavior of OLED with different anodes, the OLED with an IZTO anode shows slightly higher luminance than that of an OLED with a reference ITO anode. Even though the IZTO anode layers are prepared at room temperature, the $J$ - $V-L$ characteristics of OLEDs fabricated on amorphous IZTO anode layers are comparable to those of OLEDs fabricated on reference ITO anodes. As expected from $J-V$ curve in Fig. 7a, luminance of the OLED fabricated on a-IZTO is also slightly lower than that of OLED fabricated on reference ITO anode at the high-voltage region due to trap-limited space charge effects. ${ }^{23-26}$

Both the external quantum efficiency (EQE) and power efficiency (PE) of OLEDs fabricated on the IZTO and reference ITO anode films are shown in Fig. 8. It is noteworthy that the EQE and PE of the OLEDs with an IZTO anode are much higher than those of OLEDs with a reference ITO anode. In particular, a maximum EQE of $18.6 \%$ and a PE of $38.3 \mathrm{Lm} / \mathrm{W}$ were achieved from the phosphorescent OLED fabricated on an IZTO anode layer with the lowest sheet resistance of $15.5 \Omega / \square$. As expected from the $J$ - $V$ curve, the EQE and PE values of the OLED with an IZTO anode are also significantly dependent on the sheet resistance of the a-IZTO anode. The phosphorescent OLED fabricated on an IZTO anode with a lower sheet resistance shows higher EQE and PE values due to enhanced carrier injection. The comparable $J-V-L$ characteristics and higher efficiencies of the phosphorescent OLED of the IZTO anode
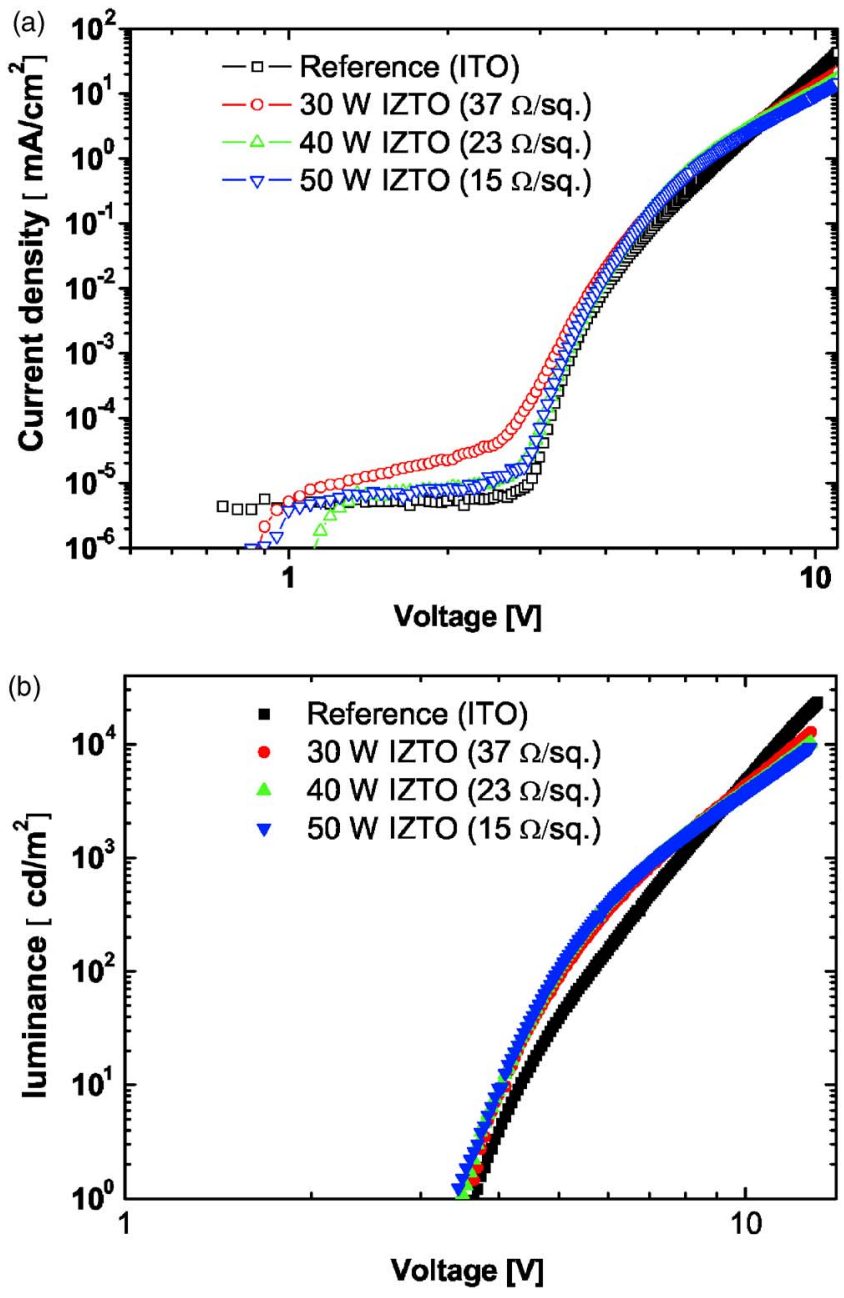

Figure 7. (Color online) (a) Current density-voltage $(J-V)$ and (b) luminance-voltage $(L-V)$ characteristics of phosphorescent OLED fabricated on an IZTO anode film as a function of sheet resistance. The reference is from an OLED with a reference ITO anode.

compared to those of the reference ITO anode can be explained by the high work function. Cui et al. ${ }^{3}$ and Marks et al. ${ }^{10}$ in investigation of the high work function transparent conducting oxide as an anode

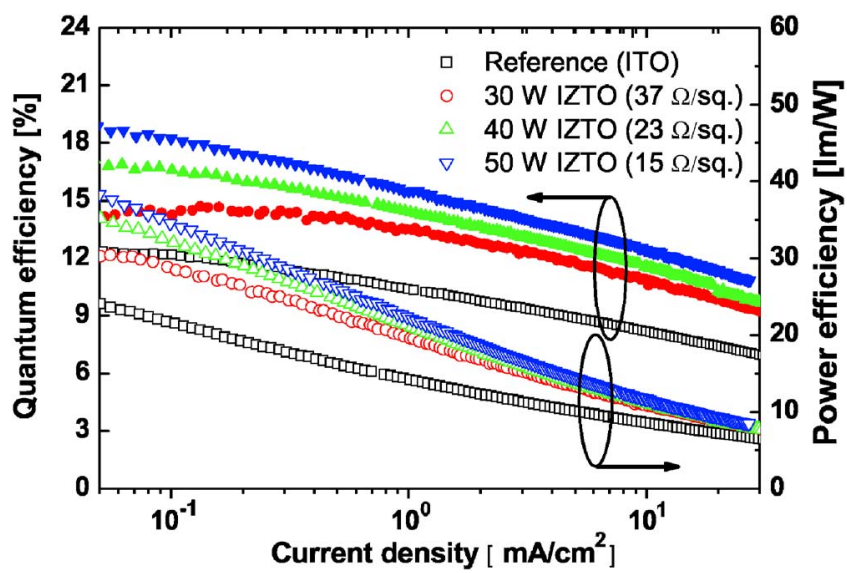

Figure 8. (Color online) External quantum efficiency and power efficiency of an OLED fabricated on an IZTO anode as a function of sheet resistance. The reference is from an OLED with a reference c-ITO anode. 
(a)

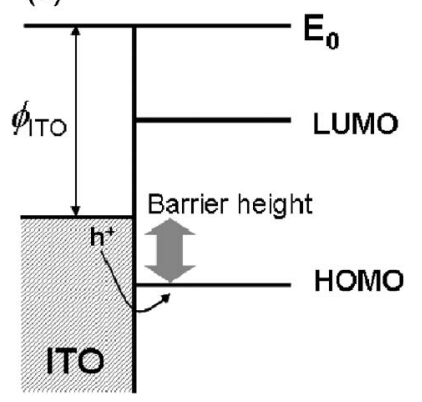

(b)

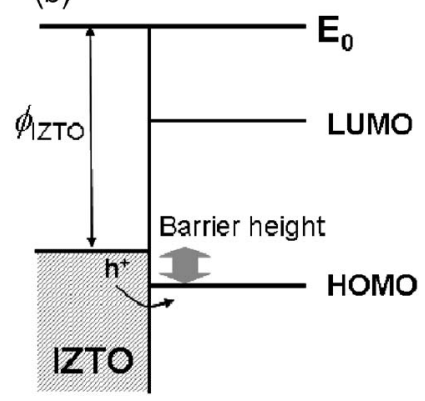

Figure 9. Schematic band diagram between anode film and organic layer. Note that anode film with a higher work function can result in more efficient hole injection from anode into the organic layer due to lower barrier height.

for an OLED, reported that an IZTO film has a higher work function $(\sim 6.1 \mathrm{eV})$ than a commercial ITO $(\sim 4.7 \mathrm{eV})$. However, the work function measured in this study from the UV ozone treated a-IZTO and reference ITO by photoelectron spectroscopy was 5.12 and $4.94 \mathrm{eV}$, respectively, a similar value to that reported by Minami et al. ${ }^{27}$ The discrepancy of the work function with the previously reported value may be caused by the difference in the stoichiometry of the IZTO target and the method of preparation. Figure 9 shows energy band diagrams of reference ITO and a-IZTO anode near the surface region. It was shown that higher work function of the a-IZTO anode than that of reference ITO anode leads to a lower barrier height between anode and organic layer. Therefore, high performance of phosphorescent OLED with IZTO anode can be attributed to the effect of high work function reducing barrier height between anode and organic layer.

\section{Conclusion}

In summary, this study has demonstrated the applicability of an IZTO anode film as an alternative to conventional ITO anode. The findings show that the high electrical conductivity, optical transmittance, and work function of the IZTO anode are comparable to those of a conventional ITO anode film even though the IZTO anode is prepared at room temperature. In addition, a phosphorescent OLED with an IZTO anode shows $J-V-L$ characteristics comparable to those of an OLED with a reference ITO anode and higher EQE and $\mathrm{PE}$ values due to the high work function of the IZTO anode. This indicates that the IZTO anode is a promising anode material that can be used as a substitute for a conventional ITO anode in OLED and flexible OLEDs.

\section{Acknowledgments}

This work was supported by a Korea Research Foundation Grant funded by the Korea Government (MOEHRD: Basic Research Promotion Fund) (grant no. KRF-2006-331-D00243) and the Ministry of Commerce, Industry, and Energy.

Kumoh National Institute of Technology assisted in meeting the publication costs of this article.

\section{References}

1. H. S. Nalwa and L. S. Rohwer, Organic Light-Emitting Diodes, American Science Publishers, Stevenson Ranch, CA (2003).

2. L. S. Hung and C. H. Chen, Mater. Sci. Eng., R., 39, 143 (2002).

3. J. Cui, A. Wang, N. L. Edleman, J. Ni, P. Lee, N. R. Armstrong, and T. Marks, Adv. Mater. (Weinheim, Ger.), 13, 1476 (2001).

4. R. Tahar, T. Ban, Y. Ohya, and Y. Takahashi, J. Appl. Phys., 83, 2631 (1998).

5. H. Kim, C. M. Gilmore, J. S. Horwitz, A. Pique, H. Murata, G. P. Kushto, R. Schlaf, Z. H. Kafafi, and D. B. Chrisey, Appl. Phys. Lett., 76, 259 (2000).

6. H. Kim, J. S. Horwitz, W. H. Kim, A. J. Makinen, Z. H. Kafafi, and D. B. Chrisey, Thin Solid Films, 420, 539 (2002).

7. J.-H. Bae, J.-M. Moon, H.-K. Kim, J.-W. Kang, H.-D. Park, J.-J. Kim, and W. J. Cho, J. Electrochem. Soc., 154, J81 (2007).

8. J. M. Phillips, R. J. Cava, G. A. Thomas, S. A. Carter, J. Kwo, T. Siegrist, J. J. Krajewski, J. H. Marshall, W. F. Peck, Jr., and D. H. Rapkine, Appl. Phys. Lett., 67, 2246 (1995).

9. A. Ambrosini, S. Malo, K. R. Poeppelmeier, M. A. Lane, C. R. Kannewurf, and T. O. Mason, Chem. Mater, 14, 58 (2002).

10. T. J. Marks, J. G. C. Veinot, J. Cui, H. Yan, A. Wang, N. L. Edlemann, J. Ni, Q. Huang, P. Lee, and N. R. Armstrong, Synth. Met., 127, 29 (2002).

11. H. M. Ali, Phys. Status Solidi A, 202, 2742 (2005).

12. N. Naghavi, C. Marcel, L. Dupont, C. Guery, C. Maugy, and J. M. Tarascon, Thin Solid Films, 419, 160 (2002).

13. T. Minami, T. Yamamoto, Y. Toda, and T. Miyata, Thin Solid Films, 373, 189 (2000).

14. J. Hsieh and C. Li, J. Appl. Phys., 42, 5295 (2003).

15. H.-C. Pan, M.-H. Shiao, C.-Y. Su, and C.-N. Hsiao, J. Vac. Sci. Technol. A, 23, 1187 (2005)

16. H. Kim, C. M. Gilmore, A. Pique, J. S. Horwitz, H. Mattoussi, H. Murata, Z. H. Kafafi, and D. B. Chrisey, J. Appl. Phys., 86, 6451 (1999).

17. G. B. Palmer, K. R. Poeppelmeier, and T. O. Mason, Chem. Mater., 9, 3121 (1997).

18. J.-H. Bae, J.-M. Moon, J.-W. Kang, H.-D. Park, J.-J. Kim, W. J. Cho, and H.-K. Kim, J. Electrochem. Soc., 154, J81 (2007).

19. J. C. C. Fan and J. B. Goodenough, J. Appl. Phys., 48, 3524 (1977).

20. G. P. Crawford, Flexible Flat Panel Displays, Wiley, New York (2005).

21. T. Tsutsui, M.-J. Yang, M. Yahiro, K. Nakamra, T. Watanabe, T. Tsuji, Y. Fukuda, T. Wakimoto, and S. Miyaguchi, Jpn. J. Appl. Phys., Part 1, 38, L1502 (1999).

22. H. J. Peng, X. L. Zhu, J. X. Sun, X. M. Yu, M. Wong, and H. S. Kwok, Appl. Phys. Lett., 88, 033509 (2006)

23. C. C. Wu, J. K. M. Chun, P. E. Burrows, J. C. Sturm, M. E. Thomson, S. R. Forrest, and R. A. Register, Appl. Phys. Lett., 66, 653 (1995).

24. P. E. Burrows and S. R. Forrest, Appl. Phys. Lett., 64, 2285 (1994).

25. Z. Chiguvare, J. Parisi, and V. Dyakonov, J. Appl. Phys., 94, 2440 (2003).

26. P. E. Burrows, Z. Shen, V. Bulovic, D. M. McCarty, S. R. Forrest, J. A. Cronin, and M. E. Thomson, J. Appl. Phys., 79, 7991 (1996).

27. T. Minami, T. Yiyata, and T. Yasmamoto, Surf. Coat. Technol., 108, 583 (1998). 